OPEN ACCESS

Edited by:

Bo Lei,

Xi'an Jiaotong University, China

Reviewed by:

Jingwei Xie,

University of Nebraska Medical

Center, United States

Chun Xu,

University of Queensland, Australia

*Correspondence:

Fang Yang

fang.yang@radboudumc.n

Specialty section:

This article was submitted to Nanoscience,

a section of the journal

Frontiers in Chemistry

Received: 24 January 2019 Accepted: 01 April 2019 Published: 25 April 2019

Citation:

Wang J, Jansen JA and Yang F (2019) Electrospraying: Possibilities and

Challenges of Engineering Carriers for

Biomedical Applications - A Mini

Review. Front. Chem. 7:258.

doi: 10.3389/fchem.2019.00258

\section{Electrospraying: Possibilities and Challenges of Engineering Carriers for Biomedical Applications-A Mini Review}

\author{
Jiamian Wang, John A. Jansen and Fang Yang* \\ Department of Biomaterials, Radboud University Medical Center, Nijmegen, Netherlands
}

Electrospraying, a liquid atomization-based technique, has been used to produce and formulate micro/nanoparticular cargo carriers for various biomedical applications, including drug delivery, biomedical imaging, implant coatings, and tissue engineering. In this mini review, we begin with the main features of electrospraying methods to engineer carriers with various bioactive cargos, including genes, growth factors, and enzymes. In particular, this review focuses on the improvement of traditional electrospraying technology for the fabrication of carriers for living cells and providing a suitable condition for gene transformation. Subsequently, the major applications of the electrosprayed carriers in the biomedical field are highlighted. Finally, we finish with conclusions and future perspectives of electrospraying for high efficiency and safe production.

Keywords: electrospraying, drug delivery, bioactive, cells, biomedical

\section{INTRODUCTION}

Electrospraying, also known as electrodynamic spraying, is capable of producing diminutive droplets with submicron sizes by means of an electric field (Khan et al., 2017). Electrospraying can also be used to produce fine polymeric particles, which are widely used for biomedical applications, particularly drug encapsulation. For the purpose of polymeric particle production, the common setup of electrospraying consists of a high-voltage power supply, a plastic/glass syringe capped by a metallic capillary (e.g., a 16- to 26-gauge needle) to hold a polymer solution, a syringe pump to control the flow of the solutions, and a grounded collector. When a high electric field is applied at the needle, a charged liquid jet will break up into droplets, which form small particles with generally narrow size distribution on the collector; the general setup can be found in Figure 1. The size and morphology of electrosprayed particles can be controlled to a certain extent by the factors related to polymer solutions [e.g., concentration, shear viscosity (Kim et al., 2015), polymer molecular weight, solvent (Bohr et al., 2015; Felice et al., 2015; Lu et al., 2015)] and electrospraying process [e.g., flow rate, electric potential difference, distance between the tip of the needle and the collector (Smeets et al., 2017)]. More details about the setup of electrospraying and its physical operating conditions can be found in other reviews (Bock et al., 2011; Ganan-Calvo et al., 2018; Jaworek et al., 2018).

Compared to the other conventional methods to produce particles, electrospraying has a few advantages, making it attractive to produce cargo carriers for biomedical applications. Firstly, the process can be performed at ambient conditions (temperature and pressure), which is beneficial for sensitive biomolecules and even living cells; secondly, due to the possible absence of an external medium that allows the dissolution or migration of water-soluble cargos, 
the encapsulation efficiency using electrospraying can be maximized (Sosnik, 2014). Thirdly, it can reproducibly provide drug-loaded nano/microparticles $(5 \mathrm{~nm}-100 \mu \mathrm{m})$ with a narrow size distribution (Chen and Pui, 1997); Coulombic repulsion between the highly charged electrosprayed droplets results in selfdispersion particles without coalescence (Xu and Hanna, 2006). Last, but not least, through adjustment of the above-mentioned solution factors and processing parameters, carriers in different structures can be obtained, such as hollow microspheres (JafariNodoushan et al., 2015; Zhou et al., 2017), nanocups (Kiran et al., 2016), porous microcarriers (Gao et al., 2015; Karimian et al., 2016; Huang et al., 2017), cell-shaped microparticles (Khanum et al., 2015; Ju et al., 2017), core-shell/multilayered microspheres (Rasekh et al., 2017; Zhang et al., 2017a), and Janus particles (Sanchez-Vazquez et al., 2017; Li et al., 2018). The schematic diagram of the structures can be found in Figure 1, and the real images can be found in the referred publications.

In the past decades, there are several comprehensive reviews published about electrospraying from different perspectives, including electrohydrodynamic technique, nature material-based systems, type of encapsulated drugs, and its applications (food, drug delivery, bone tissue engineering, etc.; Bock et al., 2012; Jayaraman et al., 2015; Arzi and Sosnik, 2018; Jacob et al., 2018; Jacobsen et al., 2018; Pawar et al., 2018; Rosell-Llompart et al., 2018). Recently, this technique continued to evolve in the field of bioengineering. Bioactive compounds, as well as cells, have been electrosprayed apart from conventional drugs. This mini review focuses on the novel bioactive cargo carriers prepared by electrospraying technique and the conditions used to maintain the bioactivity during the process. Then, we summarize the most recent development of this technology in biomedical applications regarding drug delivery, diagnostic and therapeutic biomedical imaging, implant coating, and tissue engineering. Figure $\mathbf{1}$ gives an overview of this mini review.

\section{BIOACTIVE CARGOS ELECTROSPRAYED INSIDE CARRIERS}

Due to the special character of bioactive cargos which is sensitive to the ambient environment, it has been a challenge to fabricate the bioactive cargo-loaded carrier. Here, we highlight the technical aspect of loading different bioactive cargos in terms of cells, genes, growth factors, and enzymes via the electrospraying method. Small-molecule drugs are not included in this section, which have been reviewed before (Nguyen et al., 2016).

\section{Cells}

Electrospraying has been explored to handle living cells and whole organisms (Jayasinghe, 2011), which is now known as "bioelectrospraying” (BES). BES has become an appealing tool for cell delivery in scaffolds for tissue engineering applications. BES is considered safe for cells due to the low current (usually in the nanoampere range) albeit the high voltage up to several kilovolts. Recently, a variety of cells have been electrosprayed, including fibroblasts, adipose-derived stem cells (ADSCs), periodontal ligament cells, retinal pigment epithelial cells, umbilical vascular endothelial cells, gastric epithelial cells (Xin et al., 2016), and bone marrow-derived mesenchymal stem cells (McCrea et al., 2018). The overall results demonstrated no distinct negative effect of the electrospraying process on cell vitality, morphology, and proliferation. This confirms the safety and efficiency of BES to encapsulate cells into different carriers and extracellular matrix for the study of diverse diseases.

Many polymers have been proven to be compatible with BES, including natural polymers subject to ionotropic or physical gelation (e.g., alginate, chitosan) (Qayyum et al., 2017) and some synthetic hydrogels [e.g., polyethylene glycol (PEG)]. The achieved cell encapsulation efficiency in PEG can be up to $90 \%$ (Qayyum et al., 2017). Cell aggregation was observed within the PEG hydrogel microspheres due to the lack of cell attachment site of PEG. Arg-Gly-Asp-Ser peptide-tethered PEG showed improved cell attachment, and the microencapsulated cells remained viable in the tethered PEG hydrogel microspheres for up to 7 days. Esfahani et al. (2017) encapsulated cells into semipermeable poly(lactide-co-glycolide) (PLGA) microspheres to release biologicals produced by the encapsulated cells. A coaxial system was adopted to avoid the toxicity of the solvent (chloroform/dimethylformamide) used to dissolve PLGA. Consequently, the cells and PLGA solution were only in contact at the tip of the needle before electrospraying.

BES has also been combined with electrospinning to obtain cellular tissue constructs. Yunmin et al. (2015) developed a micro integration scaffold by simultaneously electrospraying ADSCs and electrospin polyvinyl alcohol (PVA). Compared to scaffolds prepared by electrospinning a solution of ADSCs suspended in PVA, the integrated electrospraying/electrospinning one showed a larger number of surviving cells.

For further development, the BES parameters, such as cell concentration, nozzle size, polymer flow rate, voltage, and distance between the needle and the collector, need to be optimized. For example, voltage is very critical as it affects the cell vitality. Therefore, a low voltage is preferred (3-6 kV; McCrea et al., 2018). Ye et al. (2015) found that ADSCs were still viable when an electrospraying voltage of $10 \mathrm{kV}$ was used, whereas cell viability became reduced at $20 \mathrm{kV}$ and the spraying process became unstable.

In summary, BES extends the available toolkit for cell microencapsulation, and integration with other techniques, such as electrospinning, provides the possibility to form simultaneously complex living three-dimensional (3D) architectures for potential applications in regenerative medicine (Jayasinghe et al., 2015).

\section{Genes}

Gene therapy is developing rapidly as a new treatment strategy for cellular, tissue, and organ disorders, ranging from diabetes to cancer (Ward et al., 2010). To transform bacterial cells with exogenous DNA, chemo-transformation and electroporation are commonly used. However, these methods are complex and expensive, and the step of preparing competent cells is timeconsuming (Abyadeh et al., 2017). Electrospraying of DNA plasmid (pDNA) in a suitable buffer (such as high-conductivity 


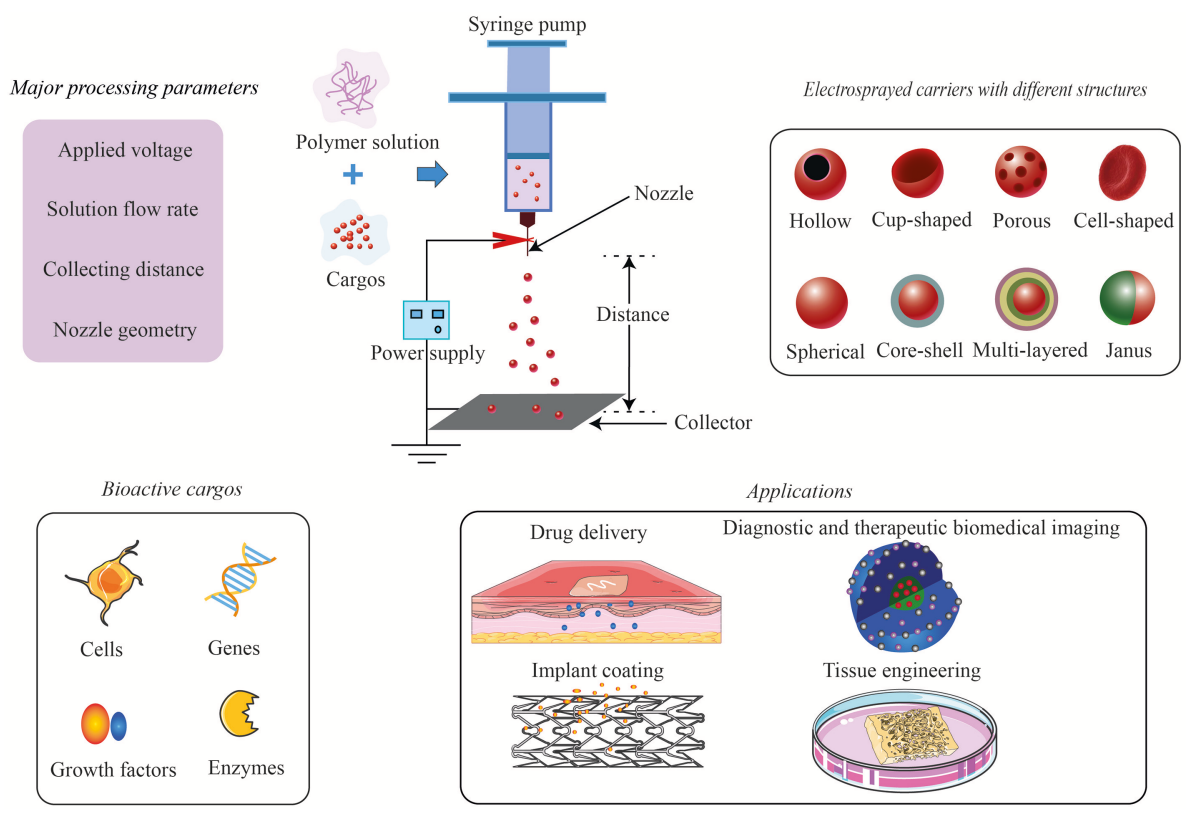

FIGURE 1 | Electrosprayed carriers: fabrication and applications.

culture medium) had been suggested as an alternative for bacterial/cell transformation with high efficiency (Boda et al., 2018). Abyadeh et al. pioneered a rapid transformation method to electrospray chitosan/pDNA into a bacterial culture to evoke a mild damage to the cell surface to increase the penetration of plasmid into bacteria. The transformation efficiency of this method was $1.7 \times 10^{8} \mathrm{CFU} / \mu \mathrm{g}$ plasmid, which is comparable to the traditional methods (Abyadeh et al., 2017).

\section{Growth Factors}

Growth factor delivery with good efficiency and efficacy is still a challenge for tissue regeneration therapies. As mentioned above, electrospraying has great benefit of reducing growth factor denaturation due to the limited exposure to organic solvents and the possibility of the dry encapsulation method (Bock et al., 2016). Zhang et al. (2017b) prepared rhBMP-2loaded PLGA microspheres with a core-shell structure via the coaxial electrospraying method and the microspheres promoted cell proliferation of bone marrow stromal cells. In order to assist growth factor bioactivity, stabilizer PEG and trehalose were added to PLGA to prepare vascular endothelial growth factor (VEGF)- or bone morphogenetic protein 7 (BMP-7)loaded carriers via electrospraying (Bock et al., 2016). Growth factor bioactivity was verified, when tested with cells, at all stages of microparticle preparation (including protein aggregation and contact with an organic solvent) and in the presence of a stabilizer in the formulation. Moreover, pre-osteoblasts (MC3T3-E1) directly co-cultured with the BMP-7-loaded carriers showed significant cell differentiation into osteoblasts. In a dual growth factor release system, double-layered microspheres were prepared by a two-step electrospraying (Xu et al., 2018). The inner layer of microspheres was first fabricated by electrospraying
BMP-2/alginic acid sodium salt solution to a dish filled with $\mathrm{CaCl}_{2}$ solution to form alginate microspheres. After being coated with chitosan, the microspheres were mixed with stromal cellderived factor-1 (SDF-1) in alginic acid sodium salt solution to go through a second electrospraying process to form the outer layer of the microspheres. The retention of bioactivities of both growth factors after the electrospraying process was confirmed.

\section{Enzymes}

A few studies explored the possibility of using electrospraying to encapsulate enzymes. Fung et al. (2016) electrosprayed coenzyme Q10 in copovidone (Kollidon ${ }^{\circledR}$ VA64) using acetone as the solvent. Both in vitro and in vivo evaluation showed enhanced bioavailability of the electrosprayed microparticles. Compared with the physical mixture of raw materials, electrosprayed microparticles revealed also enhanced dissolution properties. Yaghoobi et al. (2017) electrosprayed streptokinase-loaded PLGA nanoparticles, but found the activity of streptokinase decreased to $19.2 \%$ after electrospraying. Therefore, more research has to be done to maintain the biological activity of the enzyme during the electrospray process.

\section{APPLICATION OF THE ELECTROSPRAYED CARRIERS IN BIOMEDICINE}

Electrosprayed cargo carriers have been widely explored for various biomedical applications. In this part, the most recent applications related to electrosprayed carriers were summarized to follow the newest trend of this technique. 


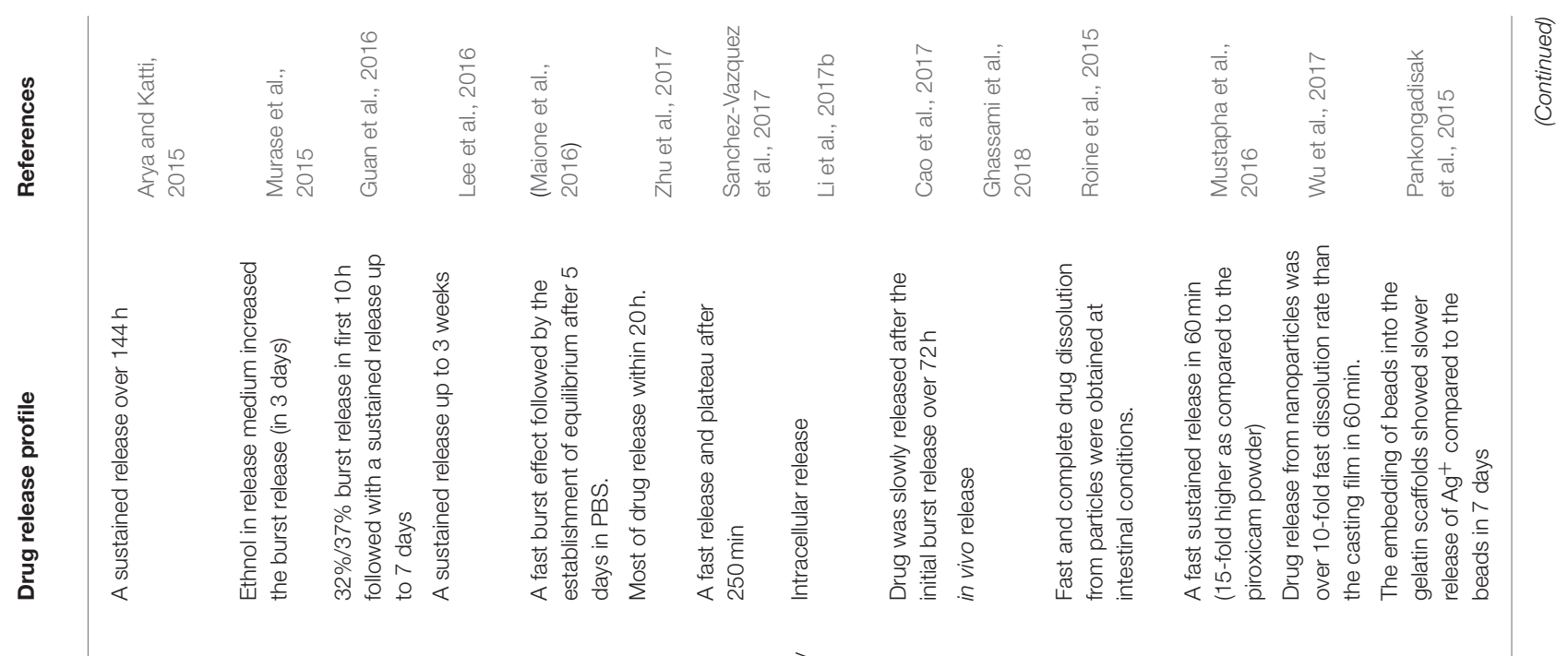

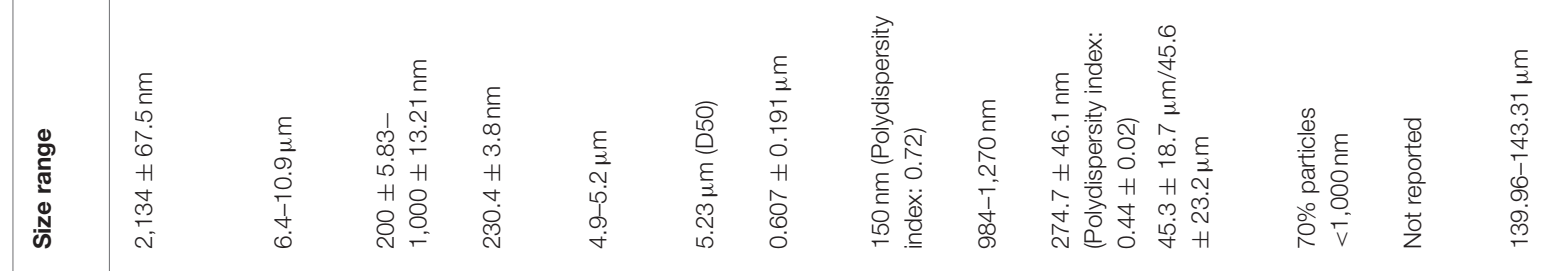
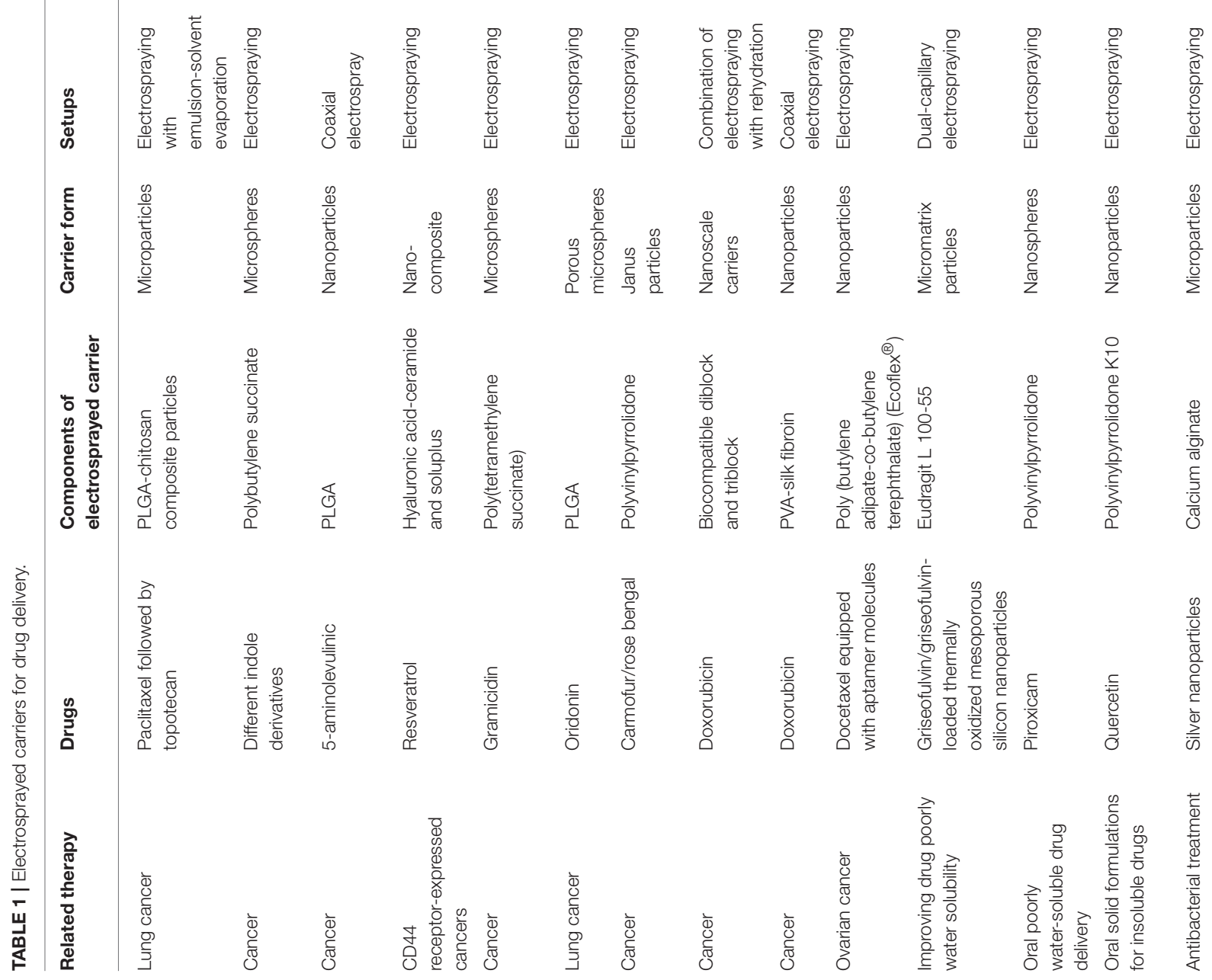


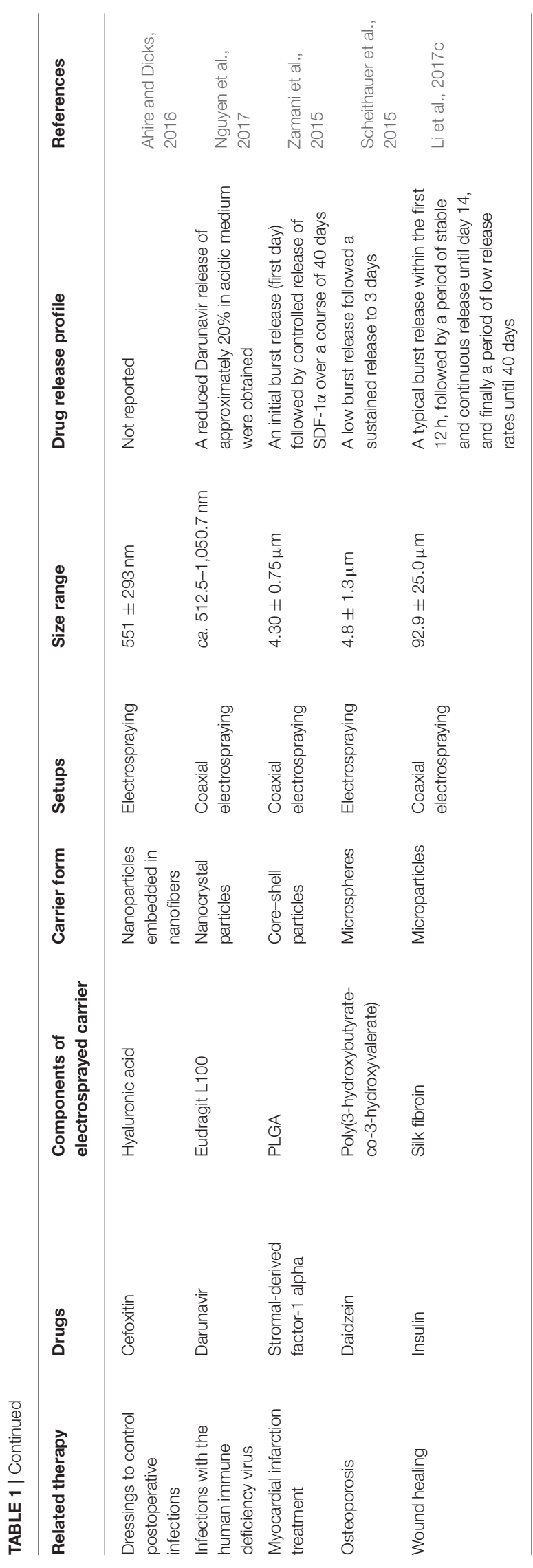

\section{Drug Delivery}

Electrospraying has been widely studied to prepare and encapsulate drugs in particles that can function as a drug delivery system. This topic has been covered by the other reviews (Nguyen et al., 2016; Boda et al., 2018; Pawar et al., 2018). In Table 1, we summarize the most recent, published from year 2015 onward, drug delivery systems developed by electrospraying to achieve particular therapeutic effects.

\section{Diagnostic and Therapeutic Biomedical Imaging}

Electrospraying has been combined with the sol-gel synthesis technique to prepare ternary phosphate-based glass nanospheres, which can be used as contrast agent for ultrasound imaging (Foroutan et al., 2015). These nanospheres are not cytotoxic at the used doses, and their function has been confirmed in vivo. The nanospheres also degrade in aqueous media, and the degradation products are easily metabolized in the body.

Taking a step ahead, Rasekh et al. (2017) applied coaxial electrospraying to encapsulate genistein (model drug), superparamagnetic iron oxide nanoparticles (10-15 nm), PEG, and a fluorescent dye in triglyceride tristearin, and demonstrated a drug release time of over $30 \mathrm{~h}$. The stable process and high drug encapsulation efficiency (around 92\%) make coaxial electrospraying a promising choice to encapsulate nanoparticles together with sensitive drugs for combined imaging and therapeutic application.

In another study, Zhang et al. (2017a) engineered particles with combined diagnostic and therapeutic functions using a trineedle coaxial electrospraying. The particles were constructed with a core-shell structure separated by an oil layer. Magnetic $\mathrm{Fe}_{3} \mathrm{O}_{4}$ nanoparticles were embedded in the polymeric shell to enable both ultrasound and magnetic resonance imaging capacity. Meanwhile, therapeutic drugs could be incorporated in both the core and the shell compartments and their release could be regulated by an external auxiliary magnetic field. A similar study was performed by electrospraying Janus particles composed of $\left[\mathrm{PLGA} / \mathrm{TbLa}_{3}(\mathrm{Bim})_{12}\right] /\left[\mathrm{PLGA} / \mathrm{Fe}_{3} \mathrm{O}_{4}\right]$ ( $\mathrm{Li}$ et al., 2018). These Janus particles showed good magnetic properties, thermal stability, biocompatibility, and enhanced fluorescent properties, displaying their potential use for biological probing and biomedical imaging.

\section{Implant Coating}

As mentioned in the Introduction, electrospraying is a versatile technique that can produce an aerosol of charged droplets with precise control of size and shape. This feature can be used to produce different morphologies of polymeric coatings on medical implants, which can guide cellular functions at the cell-implant interface. Biodegradable polyurethane with tailored microtopography was electrosprayed onto commercial coronary stents (Guo et al., 2015). The authors found the topography of coating could be manipulated by tuning the processing conditions, which influences Coulombic fission of the electrosprayed droplets. Li et al. (2017a) prepared heparin (an effective natural anticoagulant)-loaded polycaprolactone/PEG microspheres by coaxial electrospraying to coat blood vessel 
substitutes. Heparin could maintain its activity and sustained release for 15 days under the protection of the shell layer. This coating was able to prevent platelet adhesion and blood coagulation (Li et al., 2017a).

Montelukast is a selective cysLT 1 receptor antagonist and able to preserve the proliferation and migration of coronary artery endothelial cells (Zamani et al., 2016). Drug-eluting stents coated by electrosprayed montelukast-loaded PLGA was developed to inhibit the formation of neointimal hyperplasia (Zamani et al., 2016).

As the conventional polymer coatings may lose mechanical properties after implantation, novel poly (polyol sebacate)derived polymers have been explored to be used as the electrospraying coating material for metallic stents (Navarro et al., 2016). The coating can be tuned from flexible to rigid and shows no cytotoxicity on ADSCs.

\section{Tissue Engineering}

Electrosprayed particles have been widely used as biomolecule carriers for tissue regeneration, particularly bone tissue engineering. In most studies, the electrosprayed carriers were implanted into the defects or combined with a 3D scaffold. Modified coaxial electrospraying was used to prepare carriers consisting of a shell (PLGA with VEGF for angiogenesis) and core (PLGA with BMP-2 for osteogenesis; Wang et al., 2015). The obtained carriers showed an initial burst release of VEGF and a sustained release of BMP-2 with maintained bioactivity. An in vivo experiment in a rat cranial bone defect model demonstrated that growth factor-loaded spheres enhanced significantly new bone formation. In another study, nanoparticles of cartilage-specific proteins, e.g., collagen type II, hyaluronic acid, and chondroitin sulfate, were developed by electrospraying for articular cartilage repair (Yang et al., 2018). Those nanoparticles could be taken up by chondrocytes via nonspecific pinocytosis, and the gene expression of collagen type II, aggrecan, and transforming growth factor beta 1 was up-regulated, suggesting enhanced chondrocyte functionality. In another study, hydroxyapatite and calcium-deficient hydroxyapatite particles were synthesized via a sol-gel-assisted electrospraying process (Chakrapani Venkatesan et al., 2018). The calcium-deficient hydroxyapatite particles tend to display the capability to differentiate rBMSCs into the osteogenic lineage. They showed a better drug loading and release compared to the microwave-synthesized particles, with the advantages in both osteogenic differentiation and drug release for bone tissue engineering.

To enhance bone regeneration, electrospraying was combined with electrospinning to prepare a nanofibrous structured bone graft substitute. Zhu et al. (2015) used this combined fabrication method for neural tissue regeneration and engineered a highly aligned polycaprolactone microfibrous framework with embedded PLGA core-shell nanospheres for bioactive factor encapsulation. The released bioactive factor promoted rat pheochromocytoma cell proliferation and the highly aligned scaffold directed neurite extension along the fibers.

\section{CHALLENGES AND POSSIBILITIES FOR ELECTROSPRAYED CARRIERS}

Electrospraying is capable to produce fine carriers with controlled shapes/sizes and high encapsulation efficiency. It is not a complex biological or chemical modification but involves delicate engineering and material manipulation (Naqvi et al., 2016). From a technical perspective, it is a rapid, single-step approach to prepare carriers for biomedical use. However, the upscaling of this process needs a lot of investigation. To increase the preparation efficiency, Jordahl et al. (2017) invented a novel preparation process using a needleless apparatus with two parallel glass plates with narrow spaces in between $(0.35 \mathrm{~mm})$ as microchannels for electrospraying fluid. The plate edge at the outlet of the microchannels was sharpened and grooved to aid in the fluid flow. Multiple spraying jets were formed after application of electric potential, resulting in a very high production rate. In the other report, Zhang et al. (2015) described a flute-like multipore emitter device to replace conventional electrospraying capillaries for large-scale production. In BES, Zhang et al. (2016) designed a customized multihole spinneret that could produce continuous, stable jets with a five to seven times increased efficiency without affecting morphology, viability, and proliferation of human umbilical vascular endothelial cells.

Another limitation is the organic solvents used during electrospraying that may damage the bioactivity of genes, enzymes, and cell vitality. "Green electrospraying," which makes use of benign or aqueous solvents, would be the alternative to reduce toxicity (Agarwal and Greiner, 2011). Also, BES gives us an opportunity to encapsulate cells directly in scaffolds. This technique also has great potential to be used for organs-onchip, which are used to mimic the real organ to replace part of animal experiments.

In conclusion, electrospraying is a versatile technique to prepare polymeric carriers for genes, drugs, proteins, enzymes, growth factor, and cells. The application of multiple needles to improve the spraying process brings the technology closer to commercial production. Without, the use of organic solvents, green electrospraying and BES both allow the application for tissue engineering.

\section{AUTHOR CONTRIBUTIONS}

JW wrote the manuscript. JJ and FY helped to revise the manuscript.

\section{ACKNOWLEDGMENTS}

The authors acknowledge the Netherlands Organisation for Scientific Research (NWO) Domain Applied and Engineering Sciences (project number 13844) and Chinese Scholarship Council for providing the Ph.D. scholarship for JW (201506170058). 


\section{REFERENCES}

Abyadeh, M., Sadroddiny, E., Ebrahimi, A., Esmaeili, F., Landi, F. S., and Amani, A. (2017). Electrosprayed chitosan nanoparticles: facile and efficient approach for bacterial transformation. Int. Nano Lett. 7, 291-295. doi: 10.1007/s40089-017-0224-0

Agarwal, S., and Greiner, A. (2011). On the way to clean and safe electrospinninggreen electrospinning: emulsion and suspension electrospinning. Polym. Adv. Technol. 22, 372-378. doi: 10.1002/pat.1883

Ahire, J. J., and Dicks, L. M. (2016). Antimicrobial hyaluronic acid-cefoxitin sodium thin films produced by electrospraying. Curr. Microbiol. 73, 236-241. doi: $10.1007 / \mathrm{s} 00284-016-1057-1$

Arya, N., and Katti, D. S. (2015). Poly(d,l-lactide-co-glycolide)-chitosan composite particles for the treatment of lung cancer. Int. J. Nanomed. 10, 2997-3011. doi: $10.2147 /$ ijn.s78120

Arzi, R. S., and Sosnik, A. (2018). Electrohydrodynamic atomization and spraydrying for the production of pure drug nanocrystals and co-crystals. Adv. Drug Delivery Rev. 131, 79-100. doi: 10.1016/j.addr.2018.07.012

Bock, N., Dargaville, T. R., Kirby, G. T. S., Hutmacher, D. W., and Woodruff, M. A. (2016). Growth factor-loaded microparticles for tissue engineering: the discrepancies of in vitro characterization assays. Tissue Eng. Part C 22, 142-154. doi: 10.1089/ten.tec.2015.0222

Bock, N., Dargaville, T. R., and Woodruff, M. A. (2012). Electrospraying of polymers with therapeutic molecules: state of the art. Prog. Polym. Sci. 37, 1510-1551. doi: 10.1016/j.progpolymsci.2012.03.002

Bock, N., Woodruff, M. A., Hutmacher, D. W., and Dargaville, T. R. (2011). Electrospraying, a reproducible method for production of polymeric microspheres for biomedical applications. Polymers 3, 131-149. doi: 10.3390/polym 3010131

Boda, S. K., Li, X., and Xie, J. (2018). Electrospraying an enabling technology for pharmaceutical and biomedical applications: a review. J. Aerosol Sci. 125, 164-181. doi: 10.1016/j.jaerosci.2018.04.002

Bohr, A., Wan, F., Kristensen, J., Dyas, M., Stride, E., Baldursdottir, S., et al. (2015). Pharmaceutical microparticle engineering with electrospraying: the role of mixed solvent systems in particle formation and characteristics. J. Mater. Sci. Mater. Med. 26:13. doi: 10.1007/s10856-015-5379-5

Cao, Y., Liu, F. Q., Chen, Y. L., Yu, T., Lou, D. S., Guo, Y., et al. (2017). Drug release from core-shell PVA/silk fibroin nanoparticles fabricated by one-step electrospraying. Sci. Rep. 7:11913. doi: 10.1038/s41598-017-12351-1

Chakrapani Venkatesan, Y., Sampath Kumar, T. S., Raj, D. K., and Kumary, T. V. (2018). Osteogenic apatite particles by sol-gel assisted electrospraying. J. Biomed. Mater. Res. Part B 106, 1941-1954. doi: 10.1002/jbm.b.34013

Chen, D.-R., and Pui, D. Y. (1997). Experimental investigation of scaling laws for electrospraying: dielectric constant effect. Aerosol Sci. Technol. 27, 367-380. doi: $10.1080 / 02786829708965479$

Esfahani, R. R., Jun, H. S., Rahmani, S., Miller, A., and Lahann, J. (2017). Microencapsulation of live cells in synthetic polymer capsules. ACS Omega 2, 2839-2847. doi: 10.1021/acsomega.7b00570

Felice, B., Prabhakaran, M. P., Zamani, M., Rodriguez, A. P., and Ramakrishna, S. (2015). Electrosprayed poly(vinyl alcohol) particles: preparation and evaluation of their drug release profile. Polym. Int. 64, 1722-1732. doi: 10.1002/pi.4972

Foroutan, F., Jokerst, J. V., Gambhir, S. S., Vermesh, O., Kim, H. W., and Knowles, J. C. (2015). Sol-gel synthesis and electrospraying of biodegradable (P2O5)(55)$(\mathrm{CaO})(30)-(\mathrm{Na} 2 \mathrm{O})(15)$ glass nanospheres as a transient contrast agent for ultrasound stem cell imaging. ACS Nano 9, 1868-1877. doi: 10.1021/nn506789y

Fung, W. Y., Liong, M. T., and Yuen, K. H. (2016). Preparation, in-vitro and in-vivo characterisation of $\mathrm{CoQ} 10$ microparticles: electrospraying-enhanced bioavailability. J. Pharm. Pharmacol. 68, 159-169. doi: 10.1111/jphp.12502

Ganan-Calvo, A. M., Lopez-Herrera, J. M., Herrada, M. A., Ramos, A., and Montanero, J. M. (2018). Review on the physics of electrospray: from electrokinetics to the operating conditions of single and coaxial Taylor cone-jets, and AC electrospray. J. Aerosol Sci. 125, 32-56. doi: 10.1016/j.jaerosci.2018.05.002

Gao, Y., Bai, Y. T., Zhao, D., Chang, M. W., Ahmad, Z., and Li, J. S. (2015). Tuning microparticle porosity during single needle electrospraying synthesis via a non-solvent-based physicochemical approach. Polymers 7, 2701-2710. doi: 10.3390/polym7121531
Ghassami, E., Varshosaz, J., Jahanian-Najafabadi, A., Minaiyan, M., Rajabi, P., and Hayati, E. (2018). Pharmacokinetics and in vitro/in vivo antitumor efficacy of aptamer-targeted Ecoflex (R) nanoparticles for docetaxel delivery in ovarian cancer. Int. J. Nanomed. 13, 493-504. doi: 10.2147/ijn.s152474

Guan, Y. P., Zhou, Y., Sheng, J., Jiang, Z. Y., Jumahan, H., and Hu, Y. (2016), Preparation of ALA-loaded PLGA nanoparticles and its application in PDT treatment. J. Chem. Technol. Biotechnol. 91, 1128-1135. doi: 10.1002/jctb.4697

Guo, Q., Mather, J. P., Yang, P., Boden, M., and Mather, P. T. (2015). Fabrication of polymeric coatings with controlled microtopographies using an electrospraying technique. PLOS ONE 10:0129960. doi: 10.1371/journal.pone.0129960

Huang, X. W., Gao, J. F., Zheng, N., Li, W., Xue, H. G., and Li, R. K. Y. (2017). Influence of humidity and polymer additives on the morphology of hierarchically porous microspheres prepared from non-solvent assisted electrospraying. Colloids Surf. A 517, 17-24. doi: 10.1016/j.colsurfa.2017.01.003

Jacob, J., Haponiuk, J. T., Thomas, S., and Gopi, S. (2018). Biopolymer based nanomaterials in drug delivery systems: a review. Mater. Today Chem. 9, 43-55. doi: 10.1016/j.mtchem.2018.05.002

Jacobsen, C., Garcia-Moreno, P. J., Mendes, A. C., Mateiu, R. V., and Chronakis, I. S. (2018). Use of electrohydrodynamic processing for encapsulation of sensitive bioactive compounds and applications in food. Annu. Rev. Food Sci. Technol, vol. 9, 525-549. doi: 10.1146/annurev-food-030117-012348

Jafari-Nodoushan, M., Barzin, J., and Mobedi, H. (2015). Size and morphology controlling of PLGA microparticles produced by electro hydrodynamic atomization. Polym. Adv. Technol. 26, 502-513. doi: 10.1002/pat.3480

Jaworek, A., Sobczyk, A. T., and Krupa, A. (2018). Electrospray application to powder production and surface coating. J. Aerosol Sci. 125, 57-92. doi: 10.1016/j.jaerosci.2018.04.006

Jayaraman, P., Gandhimathi, C., Venugopal, J. R., Becker, D. L., Ramakrishna, S., and Srinivasan, D. K. (2015). Controlled release of drugs in electrosprayed nanoparticles for bone tissue engineering. Adv. Drug Delivery Rev. 94, 77-95. doi: 10.1016/j.addr.2015.09.007

Jayasinghe, S. N. (2011). Biojets in regenerative biology \& medicine. Mater. Today 14, 202-211. doi: 10.1016/S1369-7021(11)70115-8

Jayasinghe, S. N., Auguste, J., and Scotton, C. J. (2015). Platform technologies for directly reconstructing 3D living biomaterials. Adv. Mater. 27, 7794-7799. doi: 10.1002/adma.201503001

Jordahl, J. H., Ramcharan, S., Gregory, J. V., and Lahann, J. (2017). Needleless electrohydrodynamic cojetting of bicompartmental particles and fibers from an extended fluid interface. Macromol. Rapid Commun. 38:7. doi: $10.1002 / \operatorname{marc} .201600437$

Ju, X. J., Wang, X. X., Liu, Z., Xie, R., Wang, W., and Chu, L. Y. (2017). Red-bloodcell-shaped chitosan microparticles prepared by electrospraying. Particuology 30, 151-157. doi: 10.1016/j.partic.2016.05.011

Karimian, S. A. M., Mashayekhan, S., and Baniasadi, H. (2016). Fabrication of porous gelatin-chitosan microcarriers and modeling of process parameters via the RSM method. Int. J. Biol. Macromol. 88, 288-295. doi: 10.1016/j.ijbiomac.2016.03.061

Khan, M. K. I., Nazir, A., and Maan, A. A. (2017). Electrospraying: a novel technique for efficient coating of foods. Food Eng. Rev. 9, 112-119. doi: 10.1007/s12393-016-9150-6

Khanum, K. K., Sandeep, B. S., and Ramamurthy, P. C. (2015). Design and morphology control of a thiophene derivative through electrospraying using various solvents. RSC Adv. 5, 60419-60425. doi: 10.1039/c5ra06468f

Kim, M. K., Lee, J. Y., Oh, H., Song, D. W., Kwak, H. W., Yun, H., et al. (2015). Effect of shear viscosity on the preparation of sphere-like silk fibroin microparticles by electrospraying. Int. J. Biol. Macromol. 79, 988-995. doi: 10.1016/j.ijbiomac.2015.05.040

Kiran, A. S. K., Madhumathi, K., and Kumar, T. S. S. (2016). Electrosprayed titania nanocups for protein delivery. Colloid Interface Sci. Commun. 12:4. doi: 10.1016/j.colcom.2016.04.001

Lee, S. Y., Lee, J. J., Park, J. H., Lee, J. Y., Ko, S. H., Shim, J. S., et al. (2016). Electrosprayed nanocomposites based on hyaluronic acid derivative and Soluplus for tumor-targeted drug delivery. Colloids Surf. B 145, 267-274. doi: 10.1016/j.colsurfb.2016.05.009

Li, C. J., Wang, F. J., Ge, P., Mao, Y., and Wang, L. (2017a). Anti-acute thrombogenic surface using coaxial electrospraying coating for vascular graft application. Mater. Lett. 205, 15-19. doi: 10.1016/j.matlet.2017.06.050 
Li, K., Li, P., Jia, Z., Qi, B., Xu, J., Kang, D., et al. (2018). Enhanced fluorescent intensity of magnetic-fluorescent bifunctional PLGA microspheres based on Janus electrospraying for bioapplication. Sci. Rep. 8:17117. doi: 10.1038/s41598-018-34856-z

Li, W., Liu, S. Q., Yao, H., Liao, G. X., Si, Z. W., Gong, X. J., et al. (2017b). Microparticle templating as a route to nanoscale polymer vesicles with controlled size distribution for anticancer drug delivery. J. Colloid Interface Sci. 508, 145-153. doi: 10.1016/j.jcis.2017.08.049

Li, X., Liu, Y., Zhang, J., You, R. C., Qu, J., and Li, M. Z. (2017c). Functionalized silk fibroin dressing with topical bioactive insulin release for accelerated chronic wound healing. Mater. Sci. Eng. C Mater. Biol. Appl. 72, 394-404. doi: $10.1016 /$ j.msec.2016.11.085

Lu, J., Hou, R., Yang, Z. L., and Tang, Z. H. (2015). Development and characterization of drug-loaded biodegradable PLA microcarriers prepared by the electrospraying technique. Int. J. Mol. Med. 36, 249-254. doi: $10.3892 /$ ijmm.2015.2201

Maione, S., del Valle, L. J., Perez-Madrigal, M. M., Cativiela, C., Puiggali, J., and Aleman, C. (2016). Electrospray loading and release of hydrophobic gramicidin in polyester microparticles. RSC Adv. 6, 73045-73055. doi: 10.1039/c6ra11056h

McCrea, Z., Arnanthigo, Y., Cryan, S.-A., and O’Dea, S. (2018). A novel methodology for bio-electrospraying mesenchymal stem cells that maintains differentiation, immunomodulatory and pro-reparative functions. J. Med. Biol. Eng. 38, 497-513. doi: 10.1007/s40846-017-0331-4

Murase, S. K., Aymat, M., Calvet, A., del Valle, L. J., and Puiggali, J. (2015). Electrosprayed poly(butylene succinate) microspheres loaded with indole derivatives: a system with anticancer activity. Eur. Polym. J. 71, 196-209. doi: 10.1016/j.eurpolymj.2015.07.047

Mustapha, O., Din, F. U., Kim, D. W., Park, J. H., Woo, K. B., Lim, S. J., et al. (2016). Novel piroxicam-loaded nanospheres generated by the electrospraying technique: physicochemical characterisation and oral bioavailability evaluation. J. Microencapsulation 33, 323-330. doi: 10.1080/02652048.2016.1185475

Naqvi, S. M., Vedicherla, S., Gansau, J., McIntyre, T., Doherty, M., and Buckley, C. T. (2016). Living cell factories-electrosprayed microcapsules and microcarriers for minimally invasive delivery. Adv. Mater. 28, 5662-5671. doi: 10.1002/adma.201503598

Navarro, L., Mogosanu, D. E., de Jong, T., Bakker, A. D., Schaubroeck, D., Luna, J., et al. (2016). Poly(polyol sebacate) elastomers as coatings for metallic coronary stents. Macromol. Biosci. 16, 1678-1692. doi: 10.1002/mabi.201600105

Nguyen, D. N., Clasen, C., and Van den Mooter, G. (2016). Pharmaceutical applications of electrospraying. J. Pharm. Sci. 105, 2601-2620. doi: 10.1016/j.xphs.2016.04.024

Nguyen, D. N., Clasen, C., and Van den Mooter, G. (2017). Encapsulating darunavir nanocrystals within Eudragit L100 using coaxial electrospraying. Eur. J. Pharm. Biopharm. 113, 50-59. doi: 10.1016/j.ejpb.2016.12.002

Pankongadisak, P., Ruktanonchai, U. R., Supaphol, P., and Suwantong, O. (2015). Development of silver nanoparticles-loaded calcium alginate beads embedded in gelatin scaffolds for use as wound dressings. Polym. Int. 64, 275-283. doi: $10.1002 /$ pi. 4787

Pawar, A., Thakkar, S., and Misra, M. (2018). A bird's eye view of nanoparticles prepared by electrospraying: advancements in drug delivery field. J. Controlled Release. 286, 179-200. doi: 10.1016/j.jconrel.2018.07.036

Qayyum, A. S., Jain, E., Kolar, G., Kim, Y., Sell, S. A., and Zustiak, S. P. (2017). Design of electrohydrodynamic sprayed polyethylene glycol hydrogel microspheres for cell encapsulation. Biofabrication 9:16. doi: 10.1088/1758-5090/aa703c

Rasekh, M., Ahmad, Z., Cross, R., Hernandez-Gil, J., Wilton-Ely, J., and Miller, P. W. (2017). Facile preparation of drug-loaded tristearin encapsulated superparamagnetic iron oxide nanoparticles using coaxial electrospray processing. Mol. Pharm. 14, 2010-2023. doi: 10.1021/acs.molpharmaceut.7b00109

Roine, J., Kaasalainen, M., Peurla, M., Correia, A., Araujo, F., Santos, H. A., et al. (2015). Controlled dissolution of griseofulvin solid dispersions from electrosprayed enteric polymer micromatrix particles: physicochemical characterization and in vitro evaluation. Mol. Pharm. 12, 2254-2264. doi: $10.1021 / \mathrm{mp} 500787 \mathrm{~b}$

Rosell-Llompart, J., Grifoll, J., and Loscertales, I. G. (2018). Electrosprays in the cone-jet mode: from Taylor cone formation to spray development. J. Aerosol Sci. 125, 2-31. doi: 10.1016/j.jaerosci.2018.04.008
Sanchez-Vazquez, B., Amaral, A. J. R., Yu, D. G., Pasparakis, G., and Williams, G. R. (2017). Electrosprayed Janus particles for combined photo-chemotherapy. AAPS Pharm. Sci. Tech. 18, 1460-1468. doi: 10.1208/s12249-016-0638-4

Scheithauer, E. C., Li, W., Ding, Y. P., Harhaus, L., Roether, J. A., and Boocaccini, A. R. (2015). Preparation and characterization of electrosprayed daidzein-loaded PHBV microspheres. Mater. Lett. 158, 66-69. doi: 10.1016/j.matlet.2015.05.133

Smeets, A., Clasen, C., and Van den Mooter, G. (2017). Electrospraying of polymer solutions: study of formulation and process parameters. Eur. J. Pharm. Biopharm. 119, 114-124. doi: 10.1016/j.ejpb.2017.06.010

Sosnik, A. (2014). Production of drug-loaded polymeric nanoparticles by electrospraying technology. J. Biomed. Nanotechnol. 10, 2200-2217. doi: $10.1166 /$ jbn.2014.1887

Wang, Y., Wei, Y., Zhang, X. H., Xu, M. M., Liu, F., Ma, Q., et al. (2015). PLGA/PDLLA core-shell submicron spheres sequential release system: preparation, characterization and promotion of bone regeneration in vitro and in vivo. Chem. Eng. J. 273, 490-501. doi: 10.1016/j.cej.2015.03.068

Ward, E., Chan, E., Gustafsson, K., and Jayasinghe, S. N. (2010). Combining bio-electrospraying with gene therapy: a novel biotechnique for the delivery of genetic material via living cells. Analyst 135, 1042-1049. doi: 10.1039/b $923307 \mathrm{e}$

Wu, Y. H., Yu, D. G., Li, J. J., Wang, Q., Li, H. P., and Li, X. Y. (2017). Medicated multiple-component polymeric nanocomposites fabricated using electrospraying. Polym. Polym. Compos. 25, 57-62. doi: 10.1177/096739111702500109

Xin, Y., Chai, G., Zhang, T., Wang, X. S., Qu, M., Tan, A., et al. (2016). Analysis of multiple types of human cells subsequent to bioprinting with electrospraying technology. Biomed. Rep. 5, 723-730. doi: 10.3892/br.2016.790

Xu, C., Xu, J., Xiao, L., Li, Z., Xiao, Y., Dargusch, M., et al. (2018). Doublelayered microsphere based dual growth factor delivery system for guided bone regeneration. RSC Adv. 8, 16503-16512. doi: 10.1039/C8RA02072H

$\mathrm{Xu}, \mathrm{Y}$., and Hanna, M. A. (2006). Electrospray encapsulation of watersoluble protein with polylactide: effects of formulations on morphology, encapsulation efficiency and release profile of particles. Int. J. Pharm. 320, 30-36. doi: 10.1016/j.ijpharm.2006.03.046

Yaghoobi, N., Majidi, R. F., Faramarzi, M. A., Baharifar, H., and Amani, A. (2017). Preparation, optimization and activity evaluation of PLGA/streptokinase nanoparticles using electrospray. Adv. Pharm. Bull. 7, 131-139. doi: $10.15171 / \mathrm{apb} .2017 .017$

Yang, S. W., Ku, K. C., Chen, S. Y., Kuo, S. M., Chen, I. F., Wang, T. Y., et al. (2018). Development of chondrocyte-seeded electrosprayed nanoparticles for repair of articular cartilage defects in rabbits. J. Biomater. Appl. 32, 800-812. doi: $10.1177 / 0885328217740729$

Ye, C., He, Z. X., Lin, Y. F., Zhang, Y., Tang, J., Sun, B., et al. (2015). Bioelectrospraying is a safe technology for delivering human adipose-derived stem cells. Biotechnol. Lett. 37, 449-456. doi: 10.1007/s10529-014-1693-0

Yunmin, M., Yuanyuan, L., Haiping, C., and Qingxi, H. (2015). Application and analysis of biological electrospray in tissue engineering. Open Biomed. Eng. J. 9:133. doi: 10.2174/1874120701509010133

Zamani, M., Prabhakaran, M. P., Thian, E. S., and Ramakrishna, S. (2015). Controlled delivery of stromal derived factor- 1 alpha from poly lacticco-glycolic acid core-shell particles to recruit mesenchymal stem cells for cardiac regeneration. J. Colloid Interface Sci. 451, 144-152. doi: 10.1016/j.jcis.2015.04.005

Zamani, M., Prabhakaran, M. P., Varshosaz, J., Mhaisalkar, P. S., and Ramakrishna, S. (2016). Electrosprayed Montelukast/poly (lactic-co-glycolic acid) particle based coating: a new therapeutic approach towards the prevention of in-stent restenosis. Acta Biomater. 42, 316-328. doi: 10.1016/j.actbio.2016.07.007

Zhang, C., Yao, Z. C., Ding, Q. P., Choi, J. J., Ahmad, Z., Chang, M. W., et al. (2017a). Tri-needle coaxial electrospray engineering of magnetic polymer yolkshell particles possessing dual-imaging modality, multiagent compartments, and trigger release potential. ACS Appl. Mater. Interfaces 9, 21485-21495. doi: 10.1021/acsami.7b05580

Zhang, C. C., Chang, M. W., Ahmad, Z., Hu, W. W., Zhao, D., and Li, J. S. (2015). Stable single device multi-pore electrospraying of polymeric microparticles via controlled electrostatic interactions. RSC Adv. 5, 87919-87923. doi: 10.1039/c5ra18482g

Zhang, M., Ma, Y. L., Li, R. J., Zeng, J. H., Li, Z., Tang, Y., et al. (2017b). RhBMP-2-loaded poly(lactic-co-glycolic acid) microspheres fabricated by 
coaxial electrospraying for protein delivery. J. Biomater. Sci. Polym. Ed. 28, 2205-2219. doi: 10.1080/09205063.2017.1390381

Zhang, T., Liu, Y., Yu, H., Li, S., Chen, H., and Hu, Q. (2016). Bio-electrospraying of human umbilical vein endothelial cells with a customized multi-hole spinneret. Key Eng. Mater. 705, 291-296. doi: 10.4028/www.scientific.net/KEM. 705.291

Zhou, F. L., Chirazi, A., Gough, J. E., Cristinacce, P. L. H., and Parker, G. J. M. (2017). Hollow polycaprolactone microspheres with/without a single surface hole by co-electrospraying. Langmuir 33, 13262-13271. doi: 10.1021/acs.langmuir.7b01985

Zhu, L., Li, M., Liu, X. Y., and Jin, Y. G. (2017). Drug-loaded PLGA electrospraying porous microspheres for the local therapy of primary lung cancer via pulmonary delivery. ACS Omega 2, 2273-2279. doi: 10.1021/acsomega.7b00456

Zhu, W., Masood, F., O'Brien, J., and Zhang, L. G. (2015). Highly aligned nanocomposite scaffolds by electrospinning and electrospraying for neural tissue regeneration. Nanomed.-Nanotechnol. Biol. Med. 11, 693-704. doi: 10.1016/j.nano.2014.12.001

Conflict of Interest Statement: The authors declare that the research was conducted in the absence of any commercial or financial relationships that could be construed as a potential conflict of interest.

Copyright (c) 2019 Wang, Jansen and Yang. This is an open-access article distributed under the terms of the Creative Commons Attribution License (CC BY). The use, distribution or reproduction in other forums is permitted, provided the original author(s) and the copyright owner(s) are credited and that the original publication in this journal is cited, in accordance with accepted academic practice. No use, distribution or reproduction is permitted which does not comply with these terms. 